Vol. 13

No. 2

$79-86$

Julio - Diciembre de 2016

DOI: http://dx.doi.org/10.21676/2389783X.1723

\title{
PERCEPCIÓN DE LA CALIDAD DE ATENCIÓN RECIBIDA POR FUTUROS PADRES EN UNA CASA DE NACIMIENTOS
}

\section{PERCEIVED QUALITY OF CARE RECEIVED BY FUTURE PARENTS IN A HOUSE BIRTH}

\author{
TÍTULO CORTO: PERCEPCIÓN DE LA CALIDAD DE ATENCIÓN RECIBIDA POR \\ FUTUROS PADRES
}

\section{Esther Secanilla-Campo}

Recibido en agosto 10 de 2015

Aceptado en octubre 21 de 2015

\section{RESUMEN}

La atención a la primera infancia comienza antes de que el bebé haya nacido. En una etapa prenatal, cuanto más seguros, tranquilos y atendidos se sientan las madres y sus parejas, mejor será el proceso de parto, el postparto, la bienvenida y el desarrollo del futuro bebé. El tipo de servicio presentado atiende de una forma integral a la persona, teniendo en cuenta sus necesidades físicas, psicológicas, sociales y educativas. Este artículo aborda la evaluación de la atención profesional recibida en una casa de nacimientos, donde padres y madres deciden dar a luz a su bebé. Son centros de salud de calidad fuera del entorno hospitalario. Así mismo, se ahonda en la figura profesional de las doulas, especialistas formadas que acompañan a la mujer en el periodo primal (gestación, periodo perinatal y primer año de vida del bebé). Para ello, a partir de un registro descriptivo, se ha realizado un análisis de las respuestas de parejas atendidas $(\mathrm{n}=11)$ a un cuestionario previamente elaborado, además de realizar un estudio documental. Los resultados recogidos apuntan a la necesidad de diversificar y ofrecer centros de calidad alternativos que ofrezcan así mismo pautas de crianza durante el embarazo y después del parto, así como la necesidad de contar con un acompañamiento profesional durante todo este proceso.

Palabras clave: Calidad de vida; servicio de acompañamiento de pacientes; esperanza de vida al nacer; mujeres embarazadas; recién nacido; apego a objetos

\section{ABSTRACT}

The care to early childhood begins before the baby is born. At a prenatal phase the more care the couples feel, the better birthing process, care after childbirth and development of the baby. This service attends integrally to the person, considering the physical, psychological, social and educational needs. This article analyses the evaluation of professional attention in a health and educational centre by parents who want to give birth in quality centres outside the hospital. It also delves into the professional figure of the doula. To obtain this, from a descriptive record, it has been done the analysis of responses of couples (from a previous questioner) as a documentary analysis. The

1. Doctora en psicología de la educación. Profesora asociada del Departamento de Pedagogía sistemática y social de la Universidad Autónoma de Barcelona, España. Correo: Esther.Secanilla@uab.cat 
results suggest the need to diversify and extend to the alternative quality services affording parenting guidelines during pregnancy and after childbirth as offering career support during the whole process.

Keywords: Quality of Life; Patient Escort Service; Life Expectancy at Birth; Pregnant Women; Infant Newborn; Object Attachment

\section{INTRODUCCIÓN}

$\mathrm{E}_{\mathrm{a}}^{\mathrm{s}}$ preciso ofrecer recursos y soportes que ayuden a mejorar la calidad de vida de las personas. El servicio que se analiza en esta investigación, una casa de nacimientos, debiera considerarse como complementario a los servicios médicos ofrecidos en la actualidad, como una alternativa real de calidad donde actúan profesionales especializados y con experiencia y capacidad demostrada en su ámbito. Sobre la calidad de atención a la población hay interés desde el campo de la medicina, la psicología y las ciencias sociales, utilizando indicadores propuestos por diferentes autores ${ }^{1-8}$ para evaluar dicha calidad en centros y servicios.

Es la mujer la que decide cómo desea vivir su embarazo y su parto -recibiendo previamente la información necesaria por parte de los especialistas-, acompañada, pero no coartada, responsabilizándose libremente, pues de este modo cabe pensar que será más responsable en la crianza y en la toma de decisiones respecto a su hijo. De hecho, la participación social en salud es un recurso por medio del cual la gente juega un rol activo ${ }^{9}$. Si se parte de la importancia del proceso del embarazo y del nacimiento, hay una serie de estudios epidemiológicos que prueban la correlación entre el estado emocional de la madre durante el embarazo y aspectos tales como la sociabilidad, la agresividad o el desarrollo de la capacidad de $a m a r{ }^{10}$ durante la vida del bebé.

La percepción de los futuros padres sobre la atención de los servicios en que son atendidos es fundamental en cuanto que el espacio de confianza y de seguridad que se les ofrezca fomentará la tranquilidad a afrontar con fortaleza al nuevo ser. El objetivo principal de la casa de nacimientos analizada es precisamente ofrecer la mejor atención posible a los futuros padres y a sus hijos respetando sus deseos, intereses y emociones, acompañándolos y evitando cualquier intervención innecesaria, como la inducción al parto o la aceleración del trabajo del parto, potenciando una actitud positiva y responsable de los futuros padres, dándoles pleno protagonismo. De hecho, se debe tener en mente que cualquier paso que se de en la inducción del trabajo de parto (desprendimiento de polos, amniotomía, prostaglandinas, misoprostol, catéter de Foley, acupuntura, estimulación de los pezones, aceite de castor, etc.), implica una alta probabilidad de que hayan largas horas de oxitocina sintética, siendo un trabajo de parto inducido más difícil que un trabajo de parto que empezó espontáneamente ${ }^{11}$.

El servicio que se analiza en este estudio se sitúa en una comarca de Barcelona y está formado por matronas, ginecólogas y doulas, mujeres profesionales que acompañan a mujeres en su proceso de gestación, en el período perinatal y durante el primer año de vida. En este tipo de servicio se ofrece una atención global a la mujer durante el proceso de embarazo, el parto, el puerperio, así como durante los primeros años de vida del bebé. Se realizan visitas y pruebas obstétricas, en un clima de confianza donde se abordan los sentimientos y dificultades emocionales que comportan esta etapa. La casa de nacimientos está acondicionada con diversas salas para atender el parto, respetando la intimidad de las parejas, prestando atención a la acogida y bienvenida del recién nacido. Una vez la familia está en su domicilio, se realizan grupos de encuentro interviniendo profesionales de diferentes disciplinas pediatras, psicólogos, psicopedagogos, fisioterapeutas y enfermeras-.

No obstante, en caso de producirse una complicación durante el parto, la casa de nacimientos tiene concertado un Hospital cercano. Durante las primeras entrevistas, las parejas son asesoradas sobre el perfil necesario para ser atendidas durante el parto en la casa de nacimientos, a saber: tener embarazos considerados normales con fetos en posición cefálica y de gestación única, desear realmente optar por un parto natural, no padecer antecedentes ni médicos ni quirúrgicos que incidan de forma negativa 
durante los procesos de gestación o de parto, presentar embarazos con parto espontáneo de entre las 37 y 42 semanas. En caso de reunir las condiciones indicadas, las parejas escogen si desean ser atendidas en la casa de nacimientos o en su domicilio. Si la atención se presta en el domicilio de la pareja se verifican las condiciones, así como las vías de acceso al hospital más cercano. Si la pareja no reúne las condiciones para dar a luz en la casa de nacimientos o en el propio domicilio, igualmente recibe todos los servicios de la casa de nacimientos, aunque el parto se produzca en el hospital o clínica, acompañados siempre que sea posible por la doula de referencia.

Este tipo de servicios no cuenta con subvenciones en el estado español, siendo Holanda uno de los países europeos que aportan más experiencias. En Colombia ha habido intentos de implementar programas de salud para reducir la mortalidad materno fetal, pues es de los territorios donde se alcanzan más altas tasas de Latinoamérica. Entre otros, el modelo de atención primaria en Bogotá, modelo de atención en salud doulas para mejorar la calidad del cuidado perinatal en instituciones de primer nivel de atención ${ }^{12}$, propone atender el embarazo, parto y puerperio, además de estandarizar dicho modelo a todo el territorio colombiano para reducir la morbimortalidad. En esta propuesta citada se ofrece el diseño del modelo doula en una etapa perinatal además de difundir pautas de crianza que ofrezcan un desarrollo infantil adecuado y su implementación.

El término doula proviene del griego y significa sierva, o desde el lenguaje hindú, partera o mujer experimentada que está al servicio de otras. Son mujeres capacitadas para asistir y acompañar en el embarazo, parto y postparto a la mujer ofreciendo un apoyo emocional y psicológico. Hoy en día, en Colombia ningún hospital clínico permite la presencia de una doula en la sala de partos y solo algunos privados lo consienten. La organización Unkay funciona en Bogotá con una filosofía basada en el respeto ancestral por la naturaleza ${ }^{13}$. Cabe tener en cuenta que en todo el mundo hay una cantidad de parteras que no logran adaptarse al grado de industrialización del nacimiento. Ellas perciben que en las escuelas convencionales se les ha entrenado sobre todo para seguir protocolos establecidos por comités médicos y se sienten prisioneras de un sistema que está destruyendo el arte de la partería. Algunas buscan alternativas para la práctica en unidades obstétricas convencionales. Otras han expresado su necesidad de no ser entrenadas. Muchas de ellas se han organizado realizando encuentros a nivel internacional ${ }^{14}$. Se tiene la oportunidad de reconsiderar el fenómeno doula como figura profesional complementaria a otros especialistas.
En las últimas décadas, las organizaciones mundiales y las comunidades científicas de todo el mundo han ido adaptando protocolos y proyectos para atender el proceso de embarazo, parto y puerperio, adoptando una visión respetuosa aceptando la no intervención en la atención a la mujer y a su bebé a no ser que sea preciso. La OMS, en el año 1985 en la Declaración de Fortaleza, Brasil, realiza recomendaciones sobre las tecnologías apropiadas en el parto ${ }^{15}$ y en 1989 se publica atención efectiva durante el embarazo y el nacimiento. En la Norma 412 de 2000 se precisa la atención del parto. En el 2006 se recomienda los principios de parto integrado para el embarazo, parto y puerperio $^{16}$, y el mismo año la ley 1098 de 2006 del Código de la Infancia y la Adolescencia del Instituto Colombiano de Bienestar Familiar, en el artículo 17, Derecho de Vida y a la calidad de vida en un ambiente sano, se afirma:

\footnotetext{
Los niños, las niñas y los adolescentes tienen derecho a la vida, a una buena calidad de vida y a un ambiente sano en condiciones de dignidad y goce de todos sus derechos en forma prevalente. La calidad de vida es esencial para su desarrollo integral acorde con la dignidad del ser humano. Este derecho supone la generación de condiciones que les asegure desde la concepción cuidado, protección, alimentación nutritiva y equilibrada, acceso a los servicios de salud, educación, vestuario adecuado, recreación y vivienda segura dotada de servicios públicos esenciales en un ambiente sano. Parágrafo: El Estado desarrollará políticas públicas orientadas hacia el fortalecimiento de la primera infancia ${ }^{17}$.
}

En este sentido, ofrecer calidad en los servicios implica mejorar la calidad de vida de las personas que son atendidas; si se ofrecen recursos y soportes beneficiosos para las parejas asistidas, en el cuidado perinatal, en el parto y en el postparto, implica mejorar la calidad de vida del bebé que va a nacer.

Si se trabaja desde la prevención del bienestar de la pareja, la aceptación y la adaptación al cambio que representa el nacimiento de un hijo, hay que valorar todos los microsistemas que los envuelve ${ }^{18}$ y que implicará el desarrollo del bebé, comenzando por los conflictos que surgen en los padres individualmente y en la pareja. Pero antes está la madre como ecosistema y aún antes se encuentra el útero materno como totalidad, estando estos subsistemas en permanente influencia mutua, es el paso de un contexto seguro -la barriga de la madre- a uno nuevo, el mundo exterior, que creará inseguridades. Habitualmente se ha dicho que un bebé solo, sin su medioambiente, no existe, por eso han quedado justificados los numerosos trabajos 
realizados sobre el impacto del medioambiente sobre el niño. Si los padres perciben tanto sus competencias como el lenguaje del bebé, las interacciones padres-hijos se establecerán rápidamente ${ }^{19}$. Por tanto, según como sean estas atenciones, como vivan estos momentos, su estabilidad, sus emociones e intuiciones, influenciarán en la interrelación con su hijo. Los nueve meses del embarazo ofrecen a los futuros padres la oportunidad de prepararse tanto psicológicamente como físicamente. Efectivamente, prepararse psicológicamente implica en la mujer prepararse consciente e inconscientemente durante las etapas físicas del proceso de gestación. En el proceso psicológico del embarazo pueden manifestarse confusión o ansiedad, en este período son frecuentes el retraimiento emocional o la regresión a una actitud más dependiente respecto a otras personas de la familia. La perspectiva de aceptar y asumir la responsabilidad de un nuevo bebé crea la sensación de urgencia. El futuro progenitor necesita retraerse o experimentar una regresión con el fin de reorganizarse. La ansiedad que sienten ambos progenitores puede retrotraerlos a las disputas y a los sentimientos ambivalentes de otras adaptaciones anteriores. Esta movilización de sentimientos viejos y nuevos suministra la energía necesaria para la enorme tarea de adaptarse a un hijo. Tanto los futuros padres como quienes les atiendan (tocólogos, enfermeras, parteras) deben comprender la fuerza y la ambivalencia de los sentimientos que acompañan al embarazo ${ }^{20}$. En este sentido, la figura de la doula ofrece una amplia experiencia en este tipo de atención de tipo psicoemocional, conjuntamente con otros profesionales de la salud.

El objetivo principal de este estudio fue la evaluación del servicio dedicado al acompañamiento de los futuros padres, a partir de la percepción de una muestra de las parejas atendidas. Así mismo, se ha reflexionado entorno a la figura profesional de la doula, como agente que acompaña en el proceso de embarazo, parto y puerperio.

\section{MATERIALES Y MÉTODOS}

Se escogieron al azar once parejas (padre-madre) atendidas durante un año en la casa de nacimientos. De estas parejas, dos habían tenido anteriormente un hijo y una había tenido dos hijos.

Se partió de un registro descriptivo, se revisaron dos cuestionarios previamente diseñados por el propio servicio estudiado -uno ofrecido a las parejas antes de tener el bebé durante la primera visita al centro y un segundo cuestionario que se cumplimenta después del parto-, escogiendo los ítems relevantes para el objetivo del estudio, realizando un análisis cualitativo y cuantitativo de las respuestas de los cuestionarios y de los resultados obtenidos.

En la valoración inicial de los cuestionarios, se revisaron los indicadores de cada uno de ellos, el que se ofrece a las parejas antes de tener al bebé y el de después del parto. Se unificaron en un solo cuestionario los indicadores que resultaban adecuados para el objetivo de estudio, se valoraron la calidad y claridad de los indicadores y se rechazaron los que llevaban a confusión. Se valoró por parte de técnicos y profesionales especialistas utilizando la técnica Delphi -valoración por jueces expertos externos-, y se volvió a elaborar un único cuestionario con aquellos indicadores considerados como relevantes para la investigación.

Considerando las unidades de análisis como piezas básicas identificables en la realidad investigada y significativas para el observador mediante las cuales se percibe, se describe, se analiza y se interpreta ésta realidad ${ }^{21}$, se procedió a realizar el trabajo de campo, realizando un vaciado y análisis de la muestra recogida a partir de las trascripciones textuales, analizándolas y segmentando los datos en unidades de registro; en una siguiente fase se categorizó y analizaron los indicadores, agrupando conceptualmente fragmentos con aspectos comunes, asignándoles una denominación global, utilizando criterios de convergencia -aspectos similares, aspectos comunes- de homogeneidad interna -criterios de semejanza que tengan aspectos comunes-y de heterogeneidad externa -diferenciación externa entre categorías-. Las categorías se fueron proponiendo para cada una de las respuestas. Se codificaron asignando valores a cada respuesta, para clasificar la información. Se realizó un vaciado de las respuestas, según la categorización escogida. Finalmente se analizaron las respuestas. La valoración se hizo analizando cada madre y padre (de forma diferenciada) y después la pareja de forma global.

En la Tabla 1 se especifican las preguntas del cuestionario analizadas, una primera parte corresponde a indicadores previos al parto y la segunda a respuestas que se dan después del parto. 
Tabla 1. Preguntas del cuestionario.

\begin{tabular}{|c|c|}
\hline Antes del parto & Después del parto \\
\hline $\begin{array}{l}\text { ¿Qué sabes de tu nacimiento? } \\
\text { ¿Cómo habéis pensado recibir vuestro bebé? } \\
\text { ¿Por qué queréis tener vuestro hijo en casa o en una casa } \\
\text { de nacimientos? } \\
\text { ¿Qué esperáis de la casa de nacimientos? } \\
\text { ¿Qué piensa vuestra familia respecto a vuestra idea del } \\
\text { parto? } \\
\text { ¿Conocéis las complicaciones que pueden surgir? } \\
\text { ¿Os preocupa alguna de ellas en especial? } \\
\text { ¿Cuál sería vuestra reacción en caso de tener que ir al } \\
\text { hospital durante el parto? } \\
\text { ¿Cuál creéis que sería vuestra reacción en caso de tener un } \\
\text { problema grave con daños permanentes o muerte en casa? }\end{array}$ & $\begin{array}{l}\text { ¿Cómo habéis vivido el embarazo? } \\
\text { ¿Habéis realizado preparación al parto y al nacimiento? } \\
\text { ¿(De las respuestas afirmativas a la pregunta anterior) } \\
\text { si lo habéis realizado en una casa de nacimiento, qué } \\
\text { sugerencias harías para mejorar? } \\
\text { ¿Cómo habéis vivido el parto? } \\
\text { ¿Por qué? (respecto lo anterior) } \\
\text { ¿Os sentisteis acompañados durante el parto? } \\
\text { ¿Cómo os sentisteis acompañados? } \\
\text { ¿Habríais preferido estar solos en algún momento? } \\
\text { ¿Qué opción escogisteis para asistir a vuestro parto? } \\
\text { ¿Estáis contentos con vuestra elección? } \\
\text { ¿Si volvéis a vivir un parto repetiríais la experiencia o bien } \\
\text { cambiaríais alguna cosa? } \\
\text { ¿Por qué habéis escogido una casa de nacimientos para } \\
\text { asistir a vuestro parto? } \\
\text { ¿Cómo ha cumplido el centro vuestras expectativas y en } \\
\text { qué medida os han decepcionado? } \\
\text { ¿Cómo consideráis la ayuda de una casa de nacimiento } \\
\text { en asistiros? } \\
\text { (Respecto a lo anterior) ¿En qué se podría mejorar? }\end{array}$ \\
\hline
\end{tabular}

\section{Declaración de aspectos éticos}

A partir de las consideraciones éticas indicadas en la Declaración de Helsinki y considerando esta investigación de bajo riesgo, se solicitó el permiso de la dirección del centro para analizar los datos facilitados, dentro del centro firmando un consentimiento informado. Se informó que los resultados se utilizarían únicamente con fines de investigación y académicos. Para conseguir la confidencialidad de la información, se realizó mediante la asignación de un código a cada individuo y a cada pareja participante en el estudio, informando previamente a los participantes que sus datos serían analizados. Esta investigación se presentó al servicio estudiado, así como su análisis y propuestas.

\section{RESULTADOS}

De las respuestas obtenidas a partir de los cuestionarios analizados en su conjunto la percepción de la calidad de atención recibida en el servicio por parte de las parejas atendidas, ha sido totalmente satisfactoria. A continuación, se comentan algunos de los resultados más relevantes a partir del estudio de las respuestas categorizadas.

En cuanto a los motivos por los cuales desean tener el bebé en la casa de partos o en su propio domicilio, no dan relevancia mayoritaria al hecho de hospitalizarse, mayormente se trata de respuestas de tipo afectivo.

Las expectativas hacia los servicios de la casa de nacimientos, la totalidad de las madres desean recibir soporte emocional y profesional pero no esperan soporte educativo, en cambio los padres valoran el soporte emocional, aunque tienen mayores expectativas respecto al soporte educativo que pueda ofrecer el centro.

En cuanto a las expectativas de las familias extensas, la mayoría de parejas responde que se acepta su voluntad sin ser compartida, aun recibiendo un soporte positivo. Respecto a las complicaciones que pudieran surgir durante el parto, la mayoría de las parejas conoce las complicaciones, por lo que se deduce que las parejas toman una decisión reflexionada y están informadas. La mayoría acepta la posibilidad de ser hospitalizada si es necesario. 
Las respuestas obtenidas a los indicadores posteriores al parto, un $81 \%$ de madres ha vivido el parto como deseaba o mejor, mientras la totalidad de los padres responden que mejor. La mayoría de parejas realizaron la preparación al parto y nacimiento. De las propuestas de mejora, la mayoría de madres aportan pocas respuestas, son los padres los que solicitan más herramientas de tipo educativo en el proceso postparto, en cuanto a la crianza y seguimiento psicopedagógico en los primeros años de vida. En este sentido, se observa una cierta falta de recursos que se podría mejorar.

A la pregunta de si se sintieron acompañados durante el parto, la mayoría opina que sí, hay coincidencia de las parejas atendidas. Pero cuando responde a cómo se sintieron acompañadas, hay diferencia de respuestas entre padres y madres. La mayoría de padres no responde y los que lo hacen coinciden en afirmar que valoran el soporte afectivo, en cambio en el caso de las madres la totalidad de respuestas coincide en afirmar que donde más se sintieron acompañadas fue en la parte afectiva. El 89\% de las madres coincide en agradecer no haberse encontrado solas en ningún momento.

La mayoría de parejas estuvo atendida durante el parto en la casa de nacimientos, sólo una pareja optó por el hospital por razones médicas debido a un parto previo y otra pareja se fue de la casa de partos al hospital bajo presión familiar. Ahora bien, la mayoría de parejas llevan hasta el final su decisión. El $93 \%$ de parejas se siente muy satisfecha por la decisión tomada respecto a ser atendidos en al cas de partos. El $55 \%$ de la muestra repetiría su experiencia y el 36,4\% cambiaría algún aspecto. En cuanto a haber cumplido las expectativas sobre la casa de nacimientos, la totalidad de respuestas es afirmativa.

De los servicios que ofrece el centro, éstos tienen, según el análisis realizado, tres objetivos básicos:

- Educativos y hábitos de higiene personal: conocer el propio cuerpo, saber cómo crece el hijo, cómo evoluciona el parto, el primer vínculo afectivo con el bebé, la lactancia, vacunación, alimentación, alternativas del parto, entre otros.

84 - Sociales: reflexionar y compartir con las otras parejas los conflictos que supone el embarazo, las ilusiones, las expectativas, etc., y crear así espacios de convivencia ricos.

- Personales y Psicológicos: escucha de la propia parte intuitiva y de sentimientos, capacidades propias, confianza en el propio instinto, anatomía, importancia de la valoración del útero materno, etc.
Estos objetivos, englobados en la idea de considerar a las personas como seres sociales comunitarios, pero libres para tomar las propias decisiones.

En definitiva, las razones principales expresadas por las personas atendidas en este tipo de servicio para tener un parto natural en la casa de nacimientos o en el propio domicilio son:

- Muestra de confianza durante el proceso de gestación y en el parto

- Sentimientos de seguridad producido por un medio familiar y acompañadas por personas preparadas

- Poder recibir a sus bebés en intimidad y sin violencia

- Sentirse activas y responsables, y no meramente pacientes

- La mayoría de parejas valora muy positivamente la primera consulta realizada en el centro, que se dedica al conocimiento mutuo, se obtiene información sobre las motivaciones de las parejas que se dirigen al centro, se tratan aspectos esenciales sobre el estado de salud, antecedentes, y de otras informaciones respecto al parto y la acogida del bebé.

En este sentido, la figura de acompañamiento y apoyo ofrecida por la doula, coincidiría con muchas de las razones comentadas por las parejas estudiadas, a saber: conversa con la mujer sobre sus dudas e inquietudes respecto al parto, ofrece soporte emocional, facilita el parto trabajando conjuntamente con el resto del personal sanitario, permite que la madre tenga un entorno adecuado en el momento del parto, respeta la privacidad de la madre en todo momento, apoya al padre o hermanos 0 resto de familia extensa, asesora sobre la lactancia materna, colabora en el cuidado de la familia cuando el bebé ya está en el domicilio, aporta apoyo emocional a la madre después del parto, ofrece pautas de crianza, entre otros.

\section{DISCUSIÓN}

El hallazgo más interesante de este estudio apunta a la conclusión global por parte de las parejas analizadas, que valoran como muy positivos los servidos recibidos por parte de la casa de nacimientos donde han sido atendidos, concluyendo con una calidad satisfactoria del centro.

Se ha apuntado a la importancia que tiene atender ya desde el inicio perinatal a la infancia de forma respetuosa, así como a sus padres, para facilitar su posterior desarrollo con vínculos y espacios seguros para que se críe de forma 
rica y saludable. El hecho de atender a las familias ya desde la preparación del embarazo y el parto implica cuidar de sus hijos e hijas, que de hecho son el futuro en la sociedad. Se ha señalado la necesidad de ofrecer tanto indicadores que mejoren la propia salud de la madre, de las parejas y la de sus hijos como indicadores que procuren ofrecer estrategias psicopedagógicas a los futuros padres para mejorar sus habilidades parentales, que se corresponden con la plasticidad de los progenitores que les permite ofrecer una respuesta adecuada a las necesidades de sus hijos de acuerdo con sus fases de desarrollo ${ }^{22}$.

La importancia de ofrecer servicios donde se ofrezcan espacios seguros que permitan el establecimiento de interacciones positivas con los padres, es uno de los elementos que han quedado recogidos en este estudio. Con la atención recibida por los profesionales de este estudio y por los roles llevados a cabo por las doulas, permite que la madre y el bebé establezca un apego positivo, ofreciendo mayor seguridad emocional y permitiendo crear vínculos tanto en el entorno familiar como en otros contextos ${ }^{23}$. De hecho, si los estímulos que el entorno familiar se ofrecen son ricos, facilitadores, adecuados a las necesidades de la criatura, facilitarán el aprendizaje motor, cognitivo, lingüístico, afectivo y social del bebéé

En este sentido, sería interesante incluir propuestas que van más allá de la lactancia y crianza, propuestas que aborden por ejemplo los roles establecidos en la pareja a partir del nacimiento de un bebé, la repercusión del bebé en la vida de la pareja, las implicaciones del nacimiento del bebé en la familia extensa, entre otras. De las respuestas recogidas, se ha observado que la mayoría de padres reclaman una atención de tipo psicopedagógico una vez que se ha dado a luz, por lo que ofrecer propuestas que aborden aspectos educativos más allá de la crianza del bebé sería una línea a seguir, implementando un servicio de atención psicopedagógica.

Una vía interesante a abordar sería la de analizar el desarrollo -a nivel evolutivo, psicológico, educativo, social- de los bebés nacidos en estos contextos al cabo de un tiempo, por ejemplo: el tipo de escolarización que se escoge en el seno de la familia, el proceso de desarrollo de la autonomía, la interacción que se establece con otros iguales y con adultos, su conducta pro-social, el desarrollo de la esfera emocional e intuitiva, la salud física y mental, la evolución que como pareja se ha realizado, entre otros.
Este estudio se ha basado en los datos recogidos en una casa de nacimientos, aunque sería interesante que en futuros estudios se ampliara la muestra a otros centros que ofrecen estos servicios, tanto en la comunidad catalana y española, como en el estado de Colombia, donde existen diversos centros de esta índole. Así mismo, sería interesante poder comparar los datos recogidos sobre la calidad de los centros y realizar un estudio comparativo longitudinal entre los dos países.

Se ha analizado el valor profesional de las doulas en la atención perinatal, durante el parto y el postparto, así como en la crianza del bebé, desde la intervención, pero también desde la prevención.

\section{DECLARACIÓN SOBRE CONFLICTO DE INTERESES}

Declaro no tener ningún tipo de asociación comercial ni personal que suponga un conflicto de intereses en relación al artículo remitido. Declaro así mismo haber respetado los principios éticos en la investigación.

\section{REFERENCIAS BIBLIOGRÁFICAS}

1. Bauer R. Social Indicators. Cambridge: The M.I.T. Press; 1966.

2. Carley M. Social Measurement and Social Indicators. London: George Allen \& Unwin; 1981.

3. Land K. On the definition of social indicators. American Sociologist. 1971; 6: 322-325.

4. Gonzalez Rodriguez B. Sistemas de indicadores sociales. Madrid, I panel de expertos, Indicadores para la acción social; 1986.

5. Blanco A, Chacón F. La evaluación de la calidad de vida. En: Morales et al. Psicología social aplicada. Bilbao: Desclée de Brouwer; 1985.

6. Carmona JA. Los indicadores sociales, hoy. Madrid: Centro de investigaciones sociológicas; 1977.

7. Andrews FM. Subjective social indicators, objective social indicators and social accounting systems. En: Juster, Land. Social Accounting Systems. New York: Academic Press; 1981

8. Casas F. Técnicas de investigación social: los indicadores sociales y psicosociales. Barcelona: PPU; 1989.

9. Inuca V, Icaza L, Recalde R, Cevallos J, Romero N. Percepción de parteras y sanadores tradicionales andinos ecuatorianos en un programa de formación en salud. Duazary. 2014 Dic; 11 (2): 131-138. 
10. Rodrigáñez C. El asalto al Hades. Madrid: Proyecto editorial traficantes de sueños; 2000.

11. Odent M. iDebiéramos prohibir la Inducción del trabajo de Parto?. Midwifery Today [internet]. 2013; 107:2223. [consultado 2015 Ag 1]. Disponible en: http:// ecologiadelnacer.cl/debieramos-prohibir-la-inducciondel-trabajo-de-parto/

12. Uribe DM, Viveros L. Modelo de atención en salud "DOULAS", para mejorar la calidad del cuidado perinatal en instituciones de primer nivel de atención en Bogotá. Cuadernos Latinoamericanos de Administración. 2009; 5 (9): 95-119.

13. Archivo Digital de noticias de Colombia y El Mundo [internet]. Doulas, la reivindicación de las parteras. 2014 Abr 27; En prensa. [consultado 2015 Ag 9]. Disponible en: http://www.eltiempo.com/archivo/documento/ CMS-13888776

14. Odent M. El Granjero y el Obstetra. Buenos Aires: Creavida; 2002.

15. World Health Organization. Appropriate Technology for Birth. Lancet. 1985; 2: 436-437.

16. Organización Mundial de la Salud. Principios de parto integrado para el embarazo, parto y puerperio. 2006.
17. Colombia. Ministerio de la Protección Social. Instituto Colombiano de Bienestar Familiar. Ley 1098, por la cual se dictan disposiciones en materia de calidad de vida y atención a la primera infancia en el Código de Infancia y de Adolescencia. Capítulo II, Artículo 17: 13 (Nov 14 2006).

18. Bronfenbrenner U. La ecología del desarrollo humano. Barcelona: Ediciones Paidós; 1987.

19. Brazelton T. La naissance d'une famille ou comment se tissent les liens. Francia: Éditions Stock, Lauren Pernoud; 1983.

20. Brazelton T, Cramer B. La relación más temprana. Padres, bebés y el drama del apego inicial. Barcelona: Ediciones Paidós; 1993.

21. Evertson JM, Green JL. La observación como indagación y método. En: M.C.Wittock (ed.). La investigación en la enseñanza. II. Métodos cualitativos y de observación. Barcelona: Paidós, MEC; 1989.

22. Barudy J, Dantagnam M. Los buenos tratos a la infancia. Parentalidad, apego, resiliencia. Barcelona: Gedisa; 2005.

23. Bowlby J. Vínculos afectivos. Formación, desarrollo y pérdida. Madrid: Morata; 1995.

24. Bowlby J. El vínculo afectivo. Barcelona: Paidós; 1993.

Para citar este artículo: Secanilla-Campo E. Percepción de la calidad de atención recibida por futuros padres en una casa de nacimientos. Duazary. 2016 julio; 13 (2): 79 -86 